\title{
Bacterial tetrabromopyrrole debrominase shares a reductive dehalogenation strategy with human thyroid deiodinase
}

Jonathan R. Chekan'1 , Ga Young Lee ${ }^{2}$, Abrahim El Gamal', Trevor N. Purdy ${ }^{1}$, K. N. Houk ${ }^{2}$, and Bradley S. Moore ${ }^{1,3^{*}}$

${ }^{1}$ Center for Marine Biotechnology and Biomedicine, Scripps Institution of Oceanography, University of California San Die-go, La Jolla, CA, 92093, USA.

${ }^{2}$ Department of Chemistry and Biochemistry, University of California, Los Angeles, CA, 90095, USA.

${ }^{3}$ Skaggs School of Pharmacy and Pharmaceutical Sciences, University of California San Diego, La Jolla, CA, 92093, USA.

\section{Table of Contents}

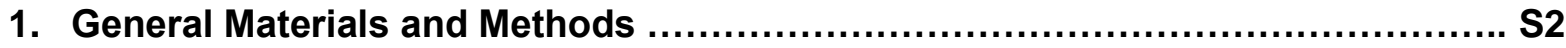

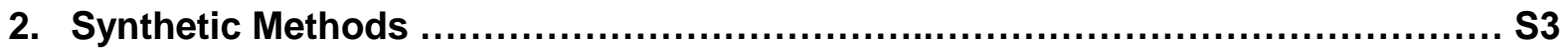

\section{Supplementary Tables}

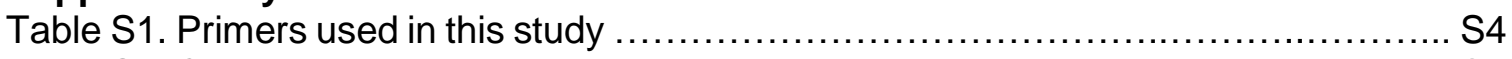

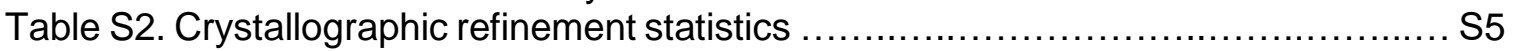

Table S3. 2,3,4,5-tetrabromopyrrole energies from B3LYP-D3 ....................... S21

Table S4. 2,3,4,5-tetrabromopyrrole energies from M06-2X ........................ S22

Table S5. Alternative substrate energies from B3LYP-D3 .......................... S25

Table S6. Dio substrate energies from B3LYP-D3 ............................. S27

\section{Supplementary Figures}

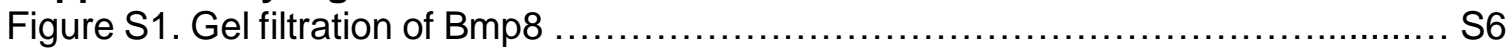

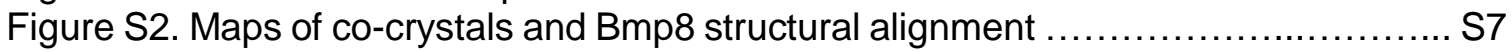

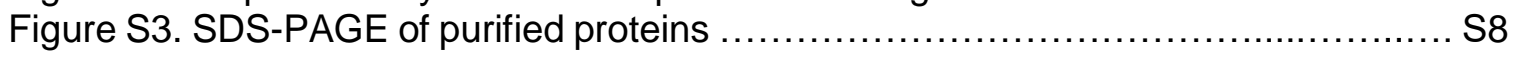

Figure S4. Calculated energetics of all possible mechanisms of tetrabromopyrrole ...... S9

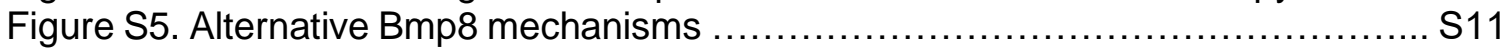

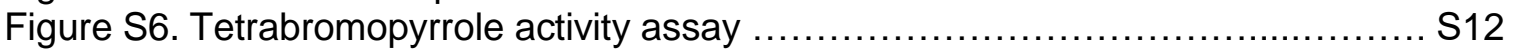

Figure S7. 2-chloro-3,4,5-tribromopyrrole activity assay ............................. 13

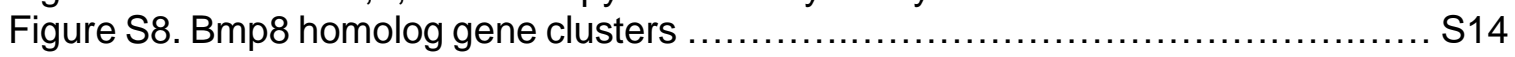

Figure S9. Bmp8 and PLCMD sequence alignment ................................. 17

\section{Computational Details}

Cartesian coordinates, energies, and vibrational frequencies 


\section{General Methods}

\section{Materials}

All materials were purchased from Fisher Scientific, Alfa Aesar, or Sigma-Aldrich. NBS and NCS were purified by recrystallization prior to use with DI water and glacial acetic acid, respectively. Preparative column chromatography was carried out on a Teledyne ISCO CombiFlash® ${ }^{\circledR} f_{+}$ Lumen $^{\mathrm{TM}}$ flash chromatography system using celite for sample loading and silica gel 60 (EMD, $40-63 \mu \mathrm{m})$ for the stationary phase. ${ }^{1} \mathrm{H}$ NMR spectra were recorded in $\mathrm{CDCl}_{3}$ (residual solvent referenced to $7.26 \mathrm{ppm}$ ) on a Jeol $500 \mathrm{MHz}$ NMR spectrometer. LCMS analysis was conducted on an Agilent 6530 Accurate-Mass Q-TOF MS (MassHunter software, Agilent) equipped with a dual electrospray ionization (ESI) source and an Agilent 1260 LC system (ChemStation software, Agilent) with diode array detector.

\section{Bacterial growth selection}

E. coli transformed with plasmids were grown in the presence of corresponding antibiotic: pET28 (kanamycin, $50 \mathrm{mg} / \mathrm{L}$ ) and pCDFDuet (spectinomycin, $50 \mathrm{mg} / \mathrm{L}$ ). 


\section{Synthetic Methods}

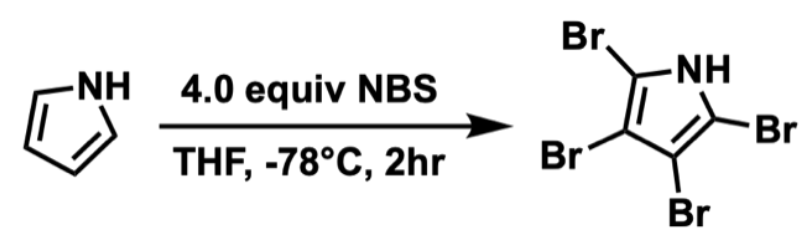

2,3,4,5-tetrabromopyrrole: This compound was synthesized using a protocol from previous work. ${ }^{1}$ A flame dried $250 \mathrm{~mL}$ round bottom flask under Ar was charged with freshly distilled pyrrole ( $0.377 \mathrm{~g}, 5.62 \mathrm{mmol}, 1.0$ equiv) and $20 \mathrm{~mL}$ dry THF. The reaction was cooled to $-78^{\circ} \mathrm{C}$ and a solution of NBS (4.00 g, $22.47 \mathrm{mmol}, 4.0$ equiv) in $65 \mathrm{~mL}$ dry THF was added dropwise via addition funnel over 30 minutes. Upon addition of NBS, the reaction was warmed to $-10^{\circ} \mathrm{C}$ for 2 hours. The reaction was then warmed to room temperature and concentrated in vacuo. The crude mixture was resuspended in EtOAc $(30 \mathrm{~mL})$ and $\mathrm{H}_{2} \mathrm{O}(25 \mathrm{~mL})$ and the aqueous layer was extracted with EtOAc $(3 \times 30 \mathrm{~mL})$. The combined organic layers were dried over $\mathrm{MgSO}_{4}$, filtered, and concentrated in vacuo to afford a white crystalline powder that slowly turned gray. Purification by flash chromatography (linear gradient $0-10 \%$ EtOAc in hexanes) and concentration under a stream of argon furnished 2,3,4,5-tetrabromopyrrole as an off-white crystalline solid $(249.7 \mathrm{mg}$, $0.652 \mathrm{mmol}, 12 \%)$. HRMS (ESI) m/z calc'd for $\mathrm{C}_{4} \mathrm{HNBr}_{4}[\mathrm{M}-\mathrm{H}]=381.6729$, found 381.6715 .<smiles></smiles>

2,3,4,5-tetrachloropyrrole: A flame dried $50 \mathrm{~mL}$ round bottom flask under Ar was charged with freshly distilled pyrrole $(0.100 \mathrm{~g}, 1.49 \mathrm{mmol}, 1.0$ equiv) and $10 \mathrm{~mL}$ dry THF. NCS $(1.19 \mathrm{~g}, 8.94$ mmol, 6.0 equiv) was directly added to the flask and the reaction was stirred at room temperature for 2 hours. Upon completion, hexanes cooled to $0^{\circ} \mathrm{C}$ was added to the reaction and the resulting solution was filtered. The filtrate was concentrated in vacuo to afford a white solid that slowly turned brown. The crude material was taken forward without further purification (119.6 mg). HRMS (ESI) m/z calc'd for $\mathrm{C}_{4} \mathrm{HNCl}_{4}[\mathrm{M}-\mathrm{H}]:$ : 203.8761, found 203.8768. 
Table S1. Primers used in this study

\begin{tabular}{|l|l|}
\hline Bmp8 M27A For & CAATTAGAGCCTGTATTTCGCGGAGCGGAACAAAATTTGGGCTTTTT \\
\hline Bmp8 M27A Rev & AAAAAGCCCAAATTTTGTTCCGCTCCGCGAAATACAGGCTCTAATTG \\
\hline Bmp8 F55A For & CGGTAGCGTTTGGTGGTTTGGCCAAATGTATCGATGCATTCA \\
\hline Bmp8 F55A Rev & TGAATGCATCGATACATTTGGCCAAACCACCAAACGCTACCG \\
\hline Bmp8 Y84F For & GCTGCATGTTTTGCAAAAGTCATTTCTCTCATATTGCGACCCGACACACGTCAA \\
\hline Bmp8 Y84F Rev & ACTTTTGCAAAACATGCAGCCCGCAGCTGAGCTACTGATCATTGCAATCGCCCAT \\
\hline Bmp8 H88V For & GCTGCATGTATTGCAAAAGTGTTTCTCTCATATTGCGACCC \\
\hline Bmp8 H88V Rev & GGGTCGCAATATGAGAGAAAACACTTTTGCAATACATGCAGC \\
\hline Bmp8 L166A For & GCTGCAATTATCGCAATTTGCGGTTTTGCAAATCGCTGGAATGCTG \\
\hline Bmp8 L166A Rev & CAGCATTCCAGCGATTTGCAAAACCGCAAATTGCGATAATTGCAGC \\
\hline Bmp8 N170A For & CGGTTTTCTAAATCGCTGGGCTGCTGCTATGGATAGTCAG \\
\hline Bmp8 N170A Rev & CTGACTATCCATAGCAGCAGCCCAGCGATTTAGAAAACCG \\
\hline Bmp8 E178Q For & GAATGCTGCTATGGATAGTCAGATACAGGCCGCTCCTAGAG \\
\hline Bmp8 E178Q Rev & CTCTAGGAGCGGCCTGTATCTGACTATCCATAGCAGCATTC \\
\hline PLCMD For & ATGCCATCATATGCCTTTAGTTACCCCTTTATCTC \\
\hline PLCMD Rev & ATGGCATCTCGAGTTAAGACTGATGTTTTCCAACTTCC \\
\hline & \\
\hline pET28 Up & CATATGGCTGCCGCGCGGCACC \\
\hline pET28 Down & CT CGAGCACCACCACCACCACCACTGAG \\
\hline
\end{tabular}


Table S2. Data collection and refinement statistics

\begin{tabular}{|c|c|c|}
\hline & Apo Bmp8 & $\begin{array}{c}\text { Bmp8 C82A } \\
\text { Tribromopyrrole Complex }\end{array}$ \\
\hline Accession code & $6 \mathrm{OHI}$ & $6 \mathrm{OHJ}$ \\
\hline \multicolumn{3}{|l|}{ Data collection } \\
\hline Space group & $P 6122$ & $P 6122$ \\
\hline \multicolumn{3}{|l|}{ Cell dimensions } \\
\hline a, b, c $(\AA)$ & $65.5,65.5,288.7$ & $65.8,65.8,288.9$ \\
\hline$\alpha, \beta, \gamma\left({ }^{\circ}\right)$ & $90.0,90.0,120.0$ & $90.0,90.0,120.0$ \\
\hline Resolution ( $(\AA)$ & $144.4-2.3(2.312-2.272)$ & $57.0-3.2(3.249-3.194)$ \\
\hline$R_{\text {sym }}{ }^{*}$ & $14.5(152.2)$ & $17.9(146.7)$ \\
\hline$R_{\mathrm{pim}}$ & $3.2(31.7)$ & $4.6(35.6)$ \\
\hline$I / \sigma l$ & $17.1(2.2)$ & $14.0(2.4)$ \\
\hline Completeness (\%) & $97.8(100)$ & $98.5(100)$ \\
\hline Redundancy & $21.9(23.7)$ & $16.2(17.5)$ \\
\hline $\mathrm{CC}_{1 / 2}$ & $0.99(0.80)$ & $0.99(0.90)$ \\
\hline \multicolumn{3}{|l|}{ Refinement } \\
\hline Resolution $(\AA ̊)$ & $56.7-2.27$ & $57.0-3.19$ \\
\hline No. reflections & 17455 & 6716 \\
\hline$R_{\text {work }} / R_{\text {free }}(\%)$ & $19.8 / 23.5$ & $23.2 / 28.2$ \\
\hline \multicolumn{3}{|l|}{ No. atoms } \\
\hline Protein & 2896 & 2906 \\
\hline Water & 59 & 2 \\
\hline \multicolumn{3}{|l|}{$B$-factors $\left(\AA^{2}\right)$} \\
\hline Protein & 45 & 95 \\
\hline Water & 44 & 82 \\
\hline Tribromopyrrole & & 207 \\
\hline \multicolumn{3}{|l|}{ R.m.s. deviations } \\
\hline Bond lengths $(\AA)$ & 0.005 & 0.003 \\
\hline Bond angles $\left({ }^{\circ}\right)$ & 1.0 & 0.5 \\
\hline
\end{tabular}

1. Highest resolution shell is shown in parenthesis.

2. $\mathrm{R}$-factor $=\Sigma\left(\left|\mathrm{F}_{\text {obs }}\right|-\mathrm{k}\left|\mathrm{F}_{\text {calc }}\right|\right) / \Sigma\left|\mathrm{F}_{\text {obs }}\right|$ and $\mathrm{R}$-free is the $\mathrm{R}$ value for a test set of reflections consisting of a random $5 \%$ of the diffraction data not used in refinement.

* Due to the high redundancy of data, $R_{\text {sym }}$ is a poor indication or quality. Instead, $C_{1 / 2}$ and $R_{\text {pim }}$ were used. 

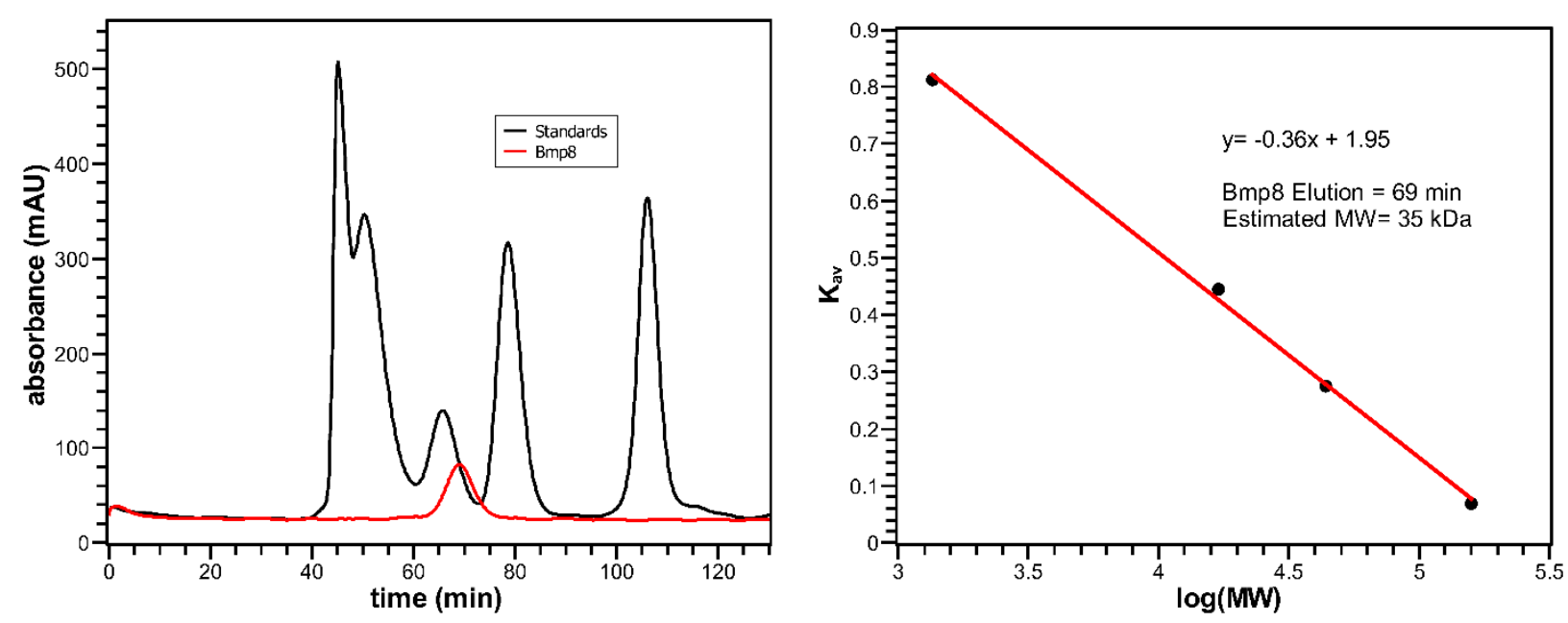

Figure S1. Gel filtration was used to estimate the size of Bmp8 in solution. A standard curve was constructed with BioRad Gel Filtration Standard (\#1511901). Bmp8 eluted at 69 mins and was estimated to be $\sim 35 \mathrm{kDa}$ in size. The molecular weight of the His-tag cut Bmp8 is 21,567 Da. The calculated complex is 1.7 monomers in size, and most likely a dimer. 


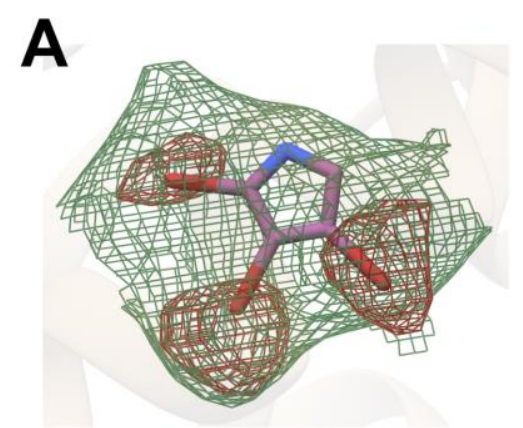

$2 \sigma F_{o}-F_{c}$

$3 \sigma$ anomolous

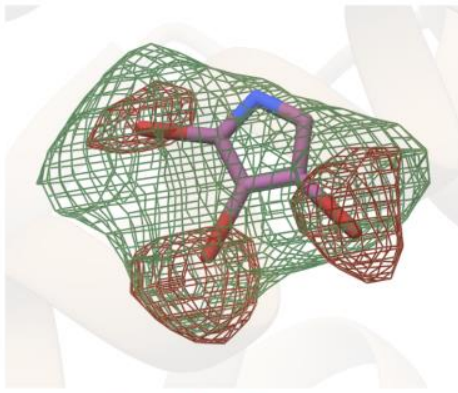

$3 \sigma \mathrm{F}_{\mathrm{o}}-\mathrm{F}_{\mathrm{c}}$

$3 \sigma$ anomolous

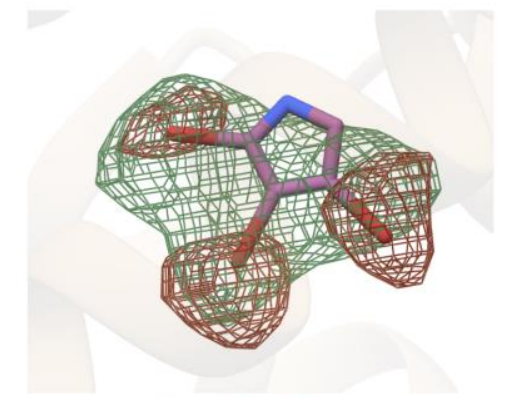

$4 \sigma F_{\mathrm{o}}-\mathrm{F}_{\mathrm{c}}$

$3 \sigma$ anomolous

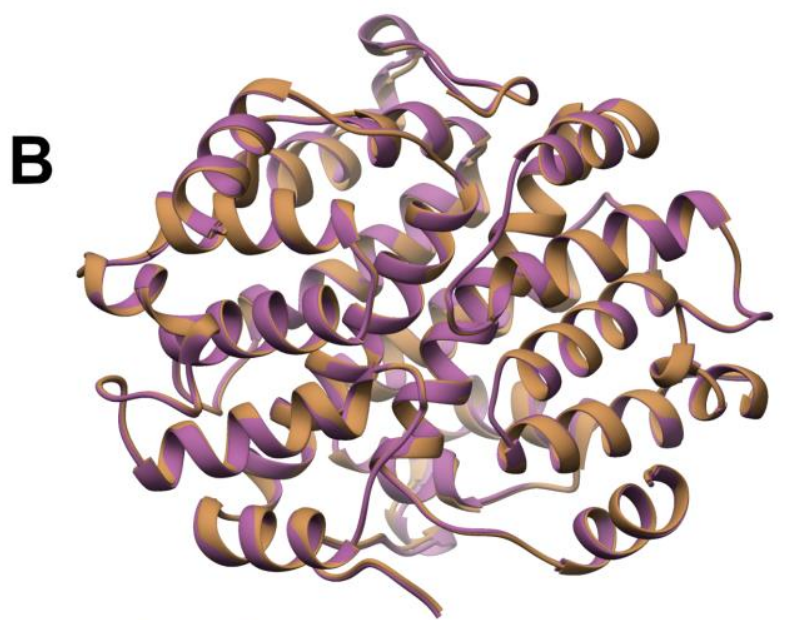

\section{Apo Bmp8}

Bmp8 C82A + Tribromopyrrole

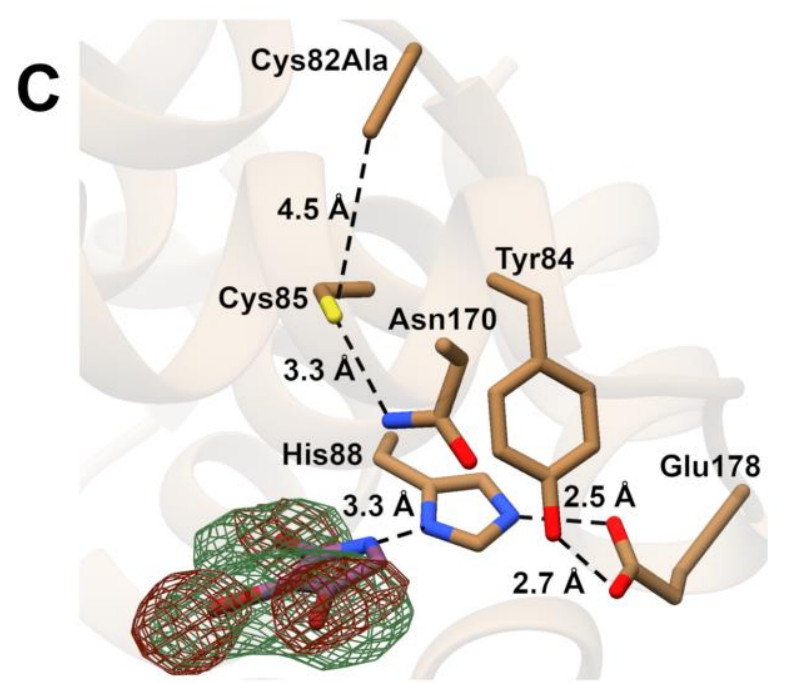

Figure S2. (A) Unhindered view of $F_{0}-F_{c}$ and anomalous maps of the Bmp8 C82A + tribromopyrrole structure refined without tribromopyrrole present. (B) Overlay with Apo Bmp8 and Bmp8 C82A + tribromopyrrole indicates that there are no notable structural movements in the presence of product. (C) Active site of Bmp8 C82A + tribromopyrrole structure with distances indicated. 


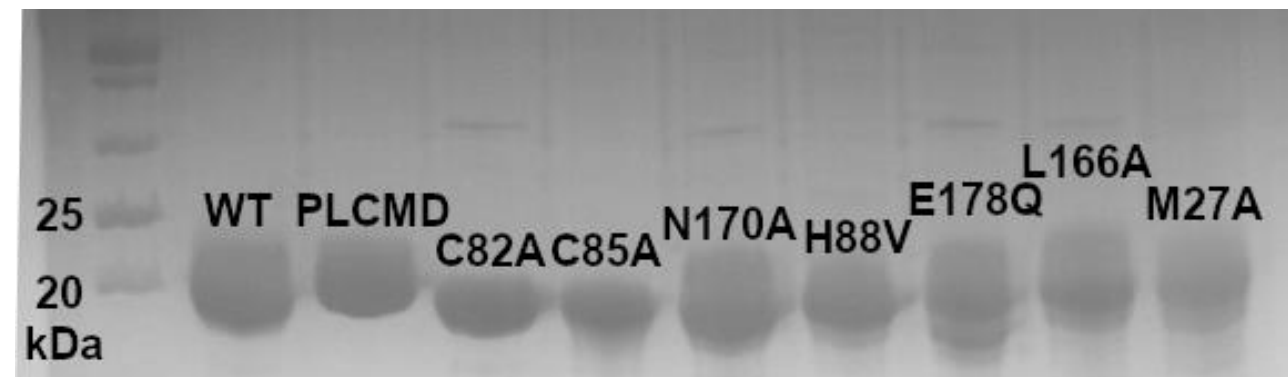

Figure S3. SDS-PAGE gel of proteins purified in this work. Fisher BioReagents EZ-Run Rec Protein Ladder was used as the marker. 


\section{Attack on sp²-hybridized C5 (SNAr)}

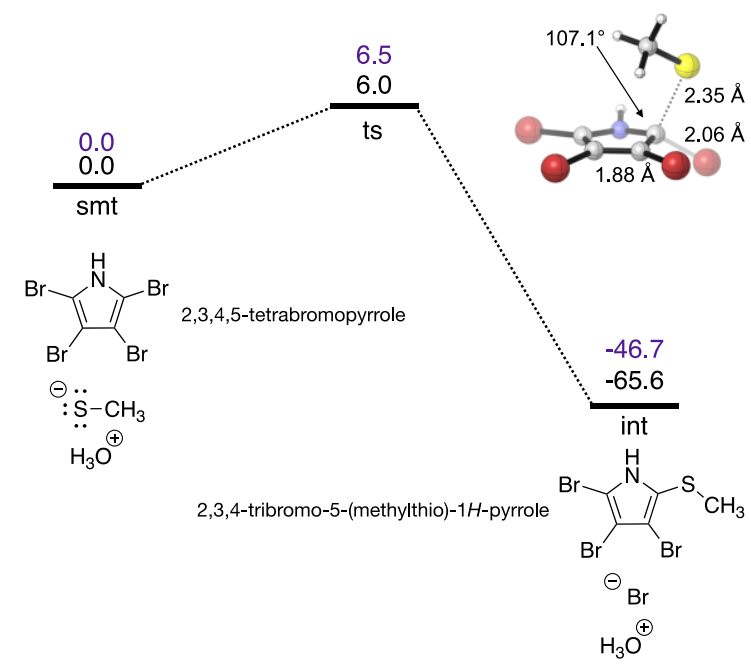

Enthalpic energies $(\Delta H)$ are in $\mathrm{kcal} / \mathrm{mol}$ and distances are in Angstrom $(\AA \AA)$

CPCM (generic,eps=4.0)/B3LYP-D3/6-311+G(d,p)/SDD

// CPCM (generic,eps=4.0)/B3LYP-D3/6-31G(d,p)/LANL2dZ

CPCM (generic,eps=4.0)/M06-2X/6-311+G(d,p) /SDD

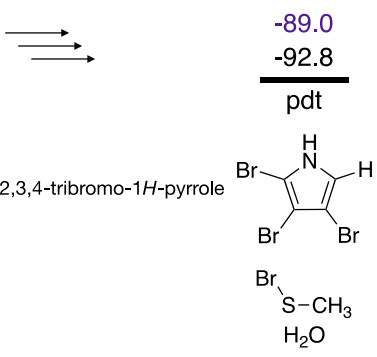

// CPCM (generic,eps=4.0)/M06-2X/6-31G(d,p)/LANL2dZ

\section{Attack on sp-hybridized C5 (SN2)}

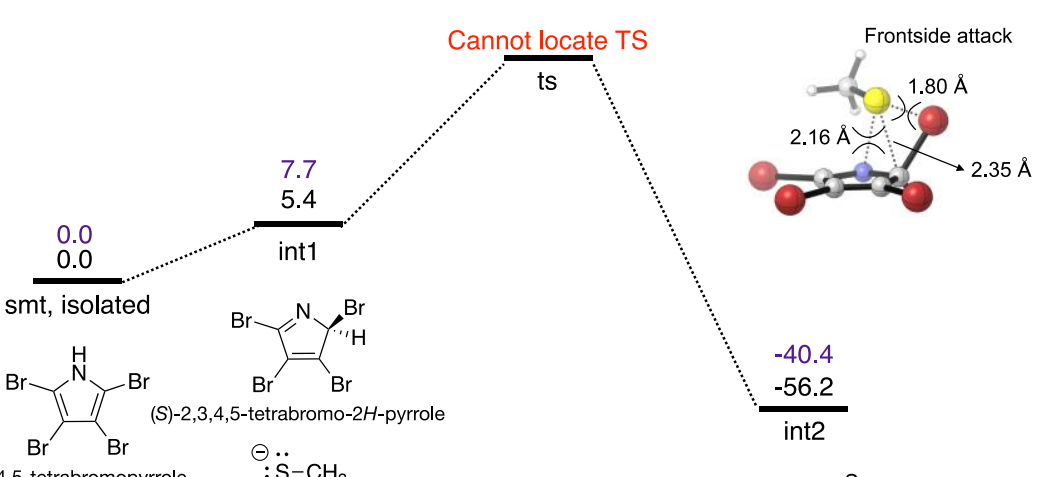

$$
\begin{aligned}
& \text { 2,3,4,5-tetrabromopyrrole } \quad: \mathrm{S}-\mathrm{CH}_{3} \\
& \stackrel{\ominus}{:}: \mathrm{S}-\mathrm{CH}_{3} \\
& \mathrm{H}_{3} \mathrm{O}^{\oplus} \\
& \mathrm{H}_{3} \mathrm{O}^{\oplus}
\end{aligned}
$$

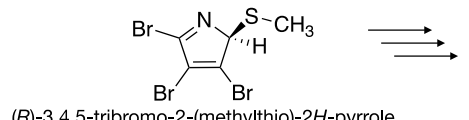

(R)-3,4,5-tribromo-2-(methylthio)-2H-pyrrole

$-92.8$

Enthalpic energies $(\Delta H)$ are in $\mathrm{kcal} / \mathrm{mol}$ and distances are in Angstrom $(\AA)$
$\mathrm{CPCM}$ (generic,eps=4.0)/B3LYP-D3/6-311+G(d,p)/SDD
// CPCM (generic,eps=4.0)/B3LYP-D3/6-31G(d,p)/LANL2dZ
$\mathrm{CPCM}$ (generic,eps=4.0)/M06-2X/6-311+G(d,p)/SDD
// CPCM (generic,eps=4.0)/M06-2X/6-31G(d,p)/LANL2dZ


Attack on $\mathrm{C5}_{\mathrm{sp} 2}-\mathrm{Br}$ (XB-based mechanism)

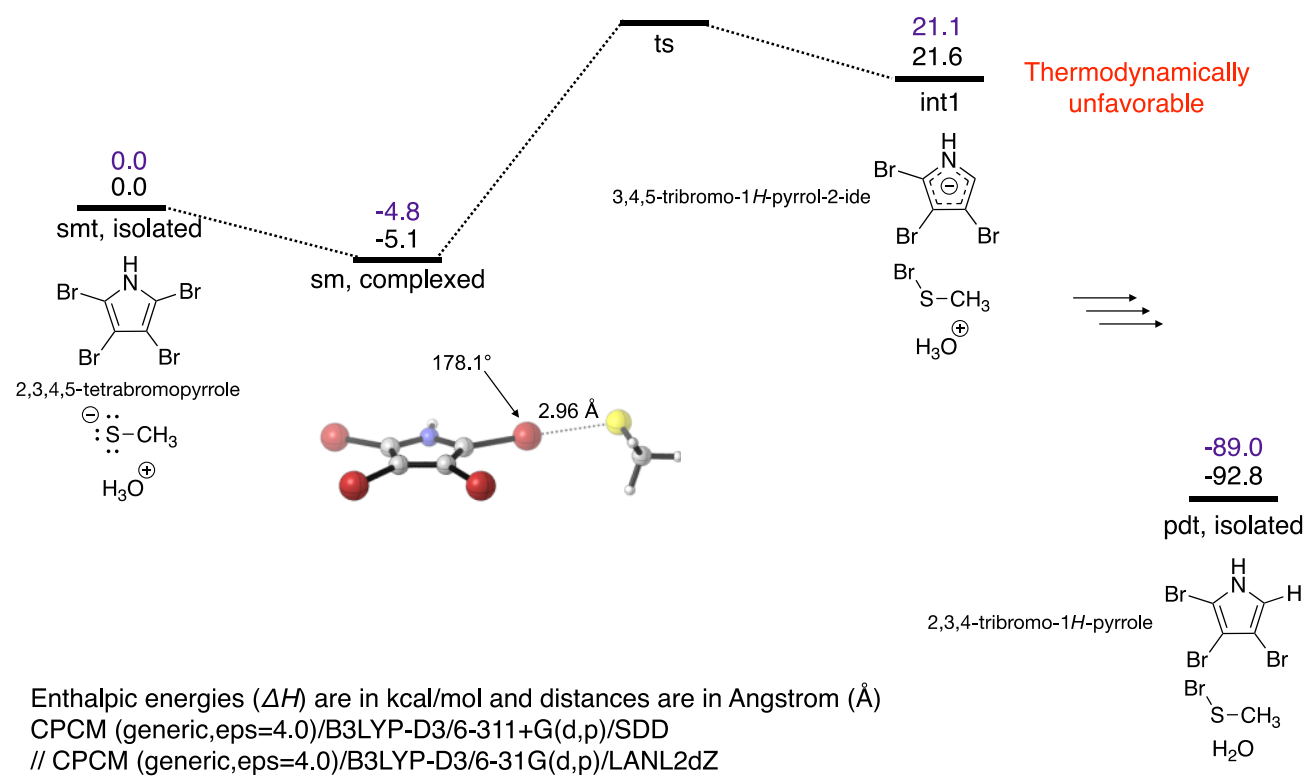

// CPCM (generic,eps=4.0)/B3LYP-D3/6-31G(d,p)/LANL2dZ

// CPCM (generic,eps=4.0)/M06-2X/6-31G(d,p)/LANL2dZ

Attack on $\mathrm{C}_{\mathrm{sp} 3}-\mathrm{Br}$ (isomerization-based mechanism)

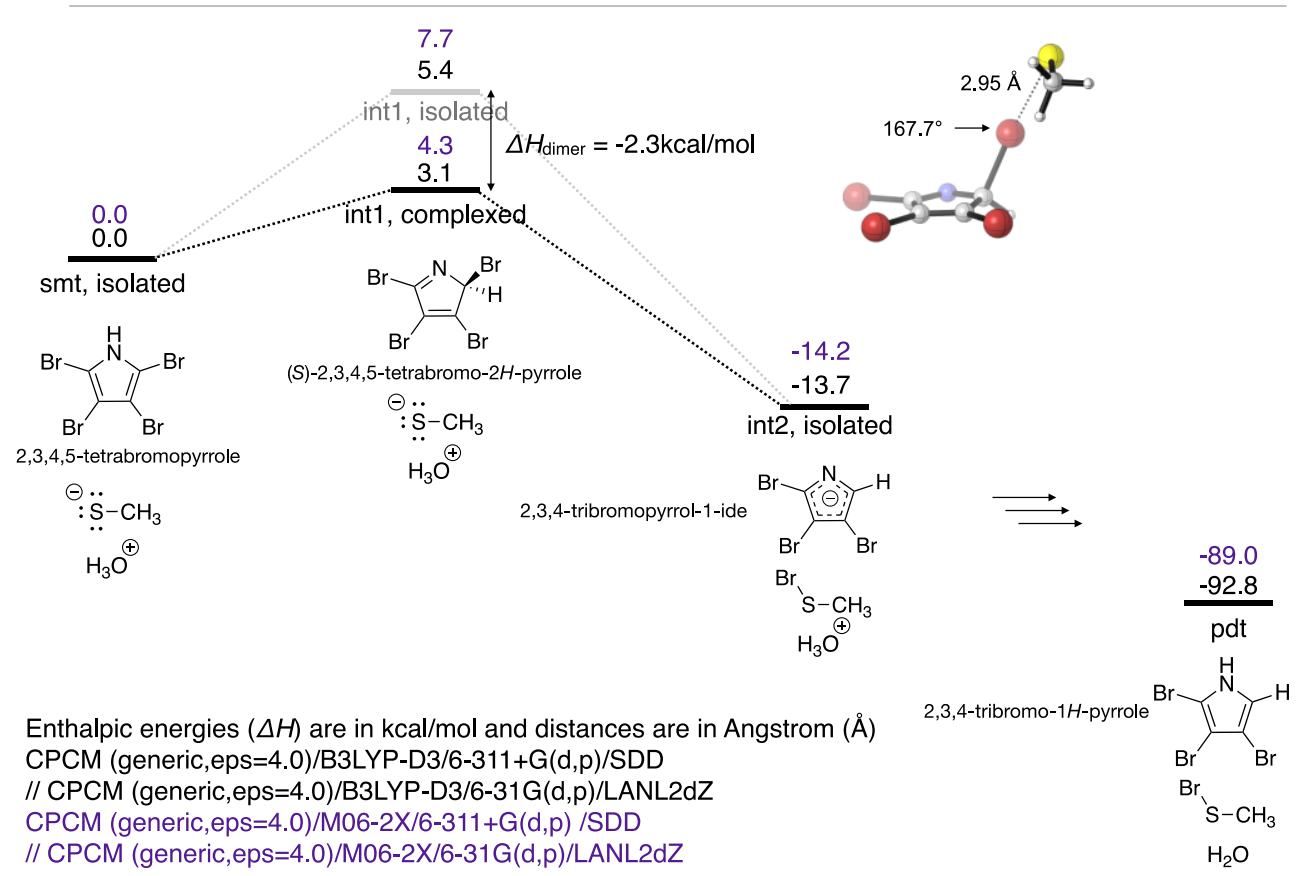

Figure S4. Calculated energetics for all possible debromination mechanisms of 2,3,4,5tetrabromopyrrole. 

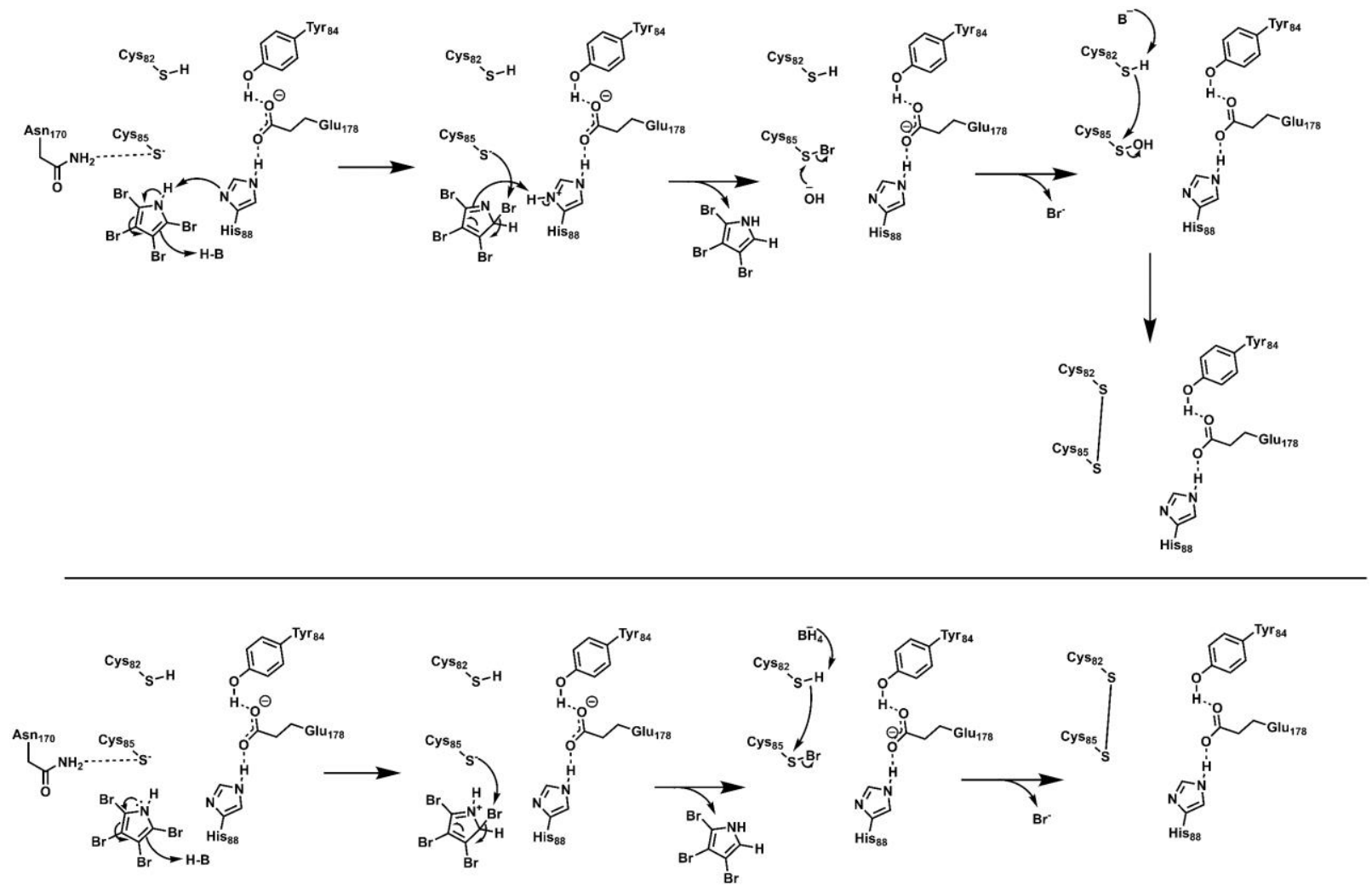

Figure S5. Alternative Bmp8 mechanisms. 

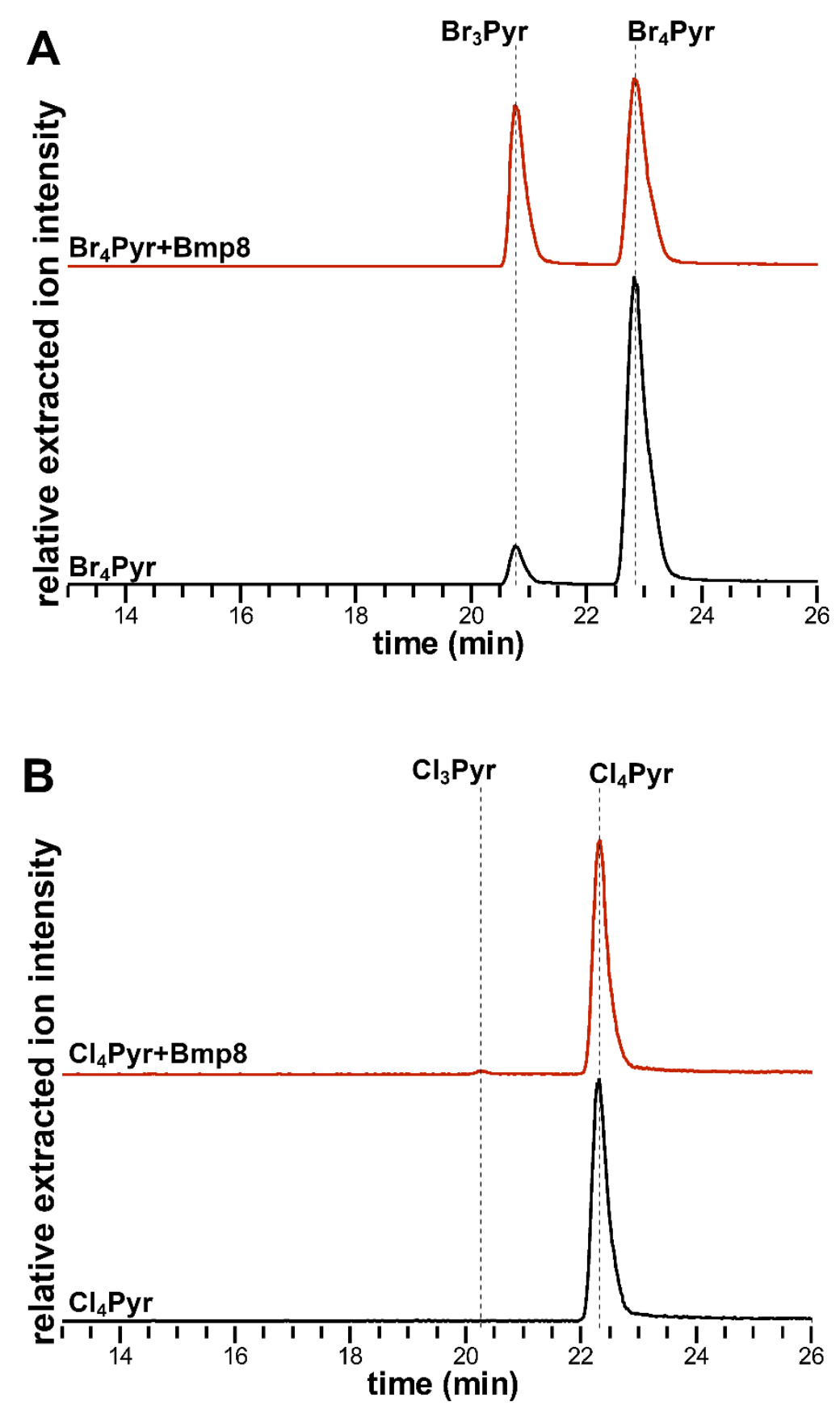

Figure S6. LCMS analysis was used to evaluate Bmp8's relative ability to dehalogenate A) tetrabromopyrrole $\left(\mathrm{Br}_{4} \mathrm{Pyr}\right)$ compared to $\left.\mathrm{B}\right)$ tetrachloropyrrole $\left(\mathrm{Cl}_{4} \mathrm{Pyr}\right)$. Traces represent the combined extracted ion chromatograms for the expected major isotopic $[\mathrm{M}-\mathrm{H}]^{-}$masses of $A$ ) $\mathrm{Br}_{4} \operatorname{Pyr}(381.7 \mathrm{~m} / \mathrm{z})$ and $\mathrm{Br}_{3} \operatorname{Pyr}(301.8 \mathrm{~m} / \mathrm{z})$ or B) $\mathrm{Cl}_{4} \operatorname{Pyr}(203.9 \mathrm{~m} / \mathrm{z})$ and $\mathrm{Cl}_{3} \mathrm{Pyr}(167.9 \mathrm{~m} / \mathrm{z})$. 

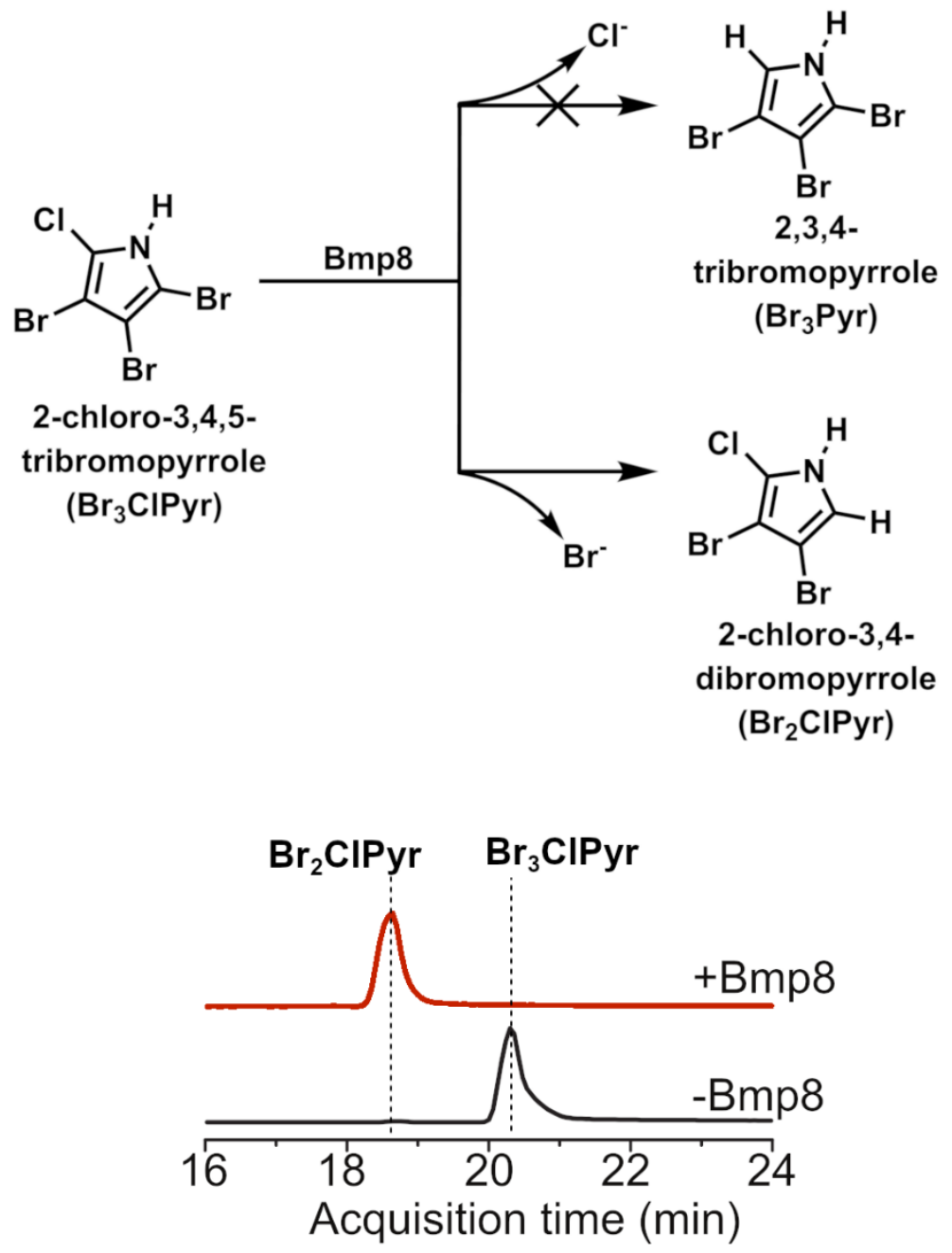

Figure S7. Bmp8 was tested for activity with 2-chloro-3,4,5-tribromopyrrole. Analysis by LCMS indicated that Bmp8 was able to debrominate the substrate, but no significant amount of dechlorinated product was detected. Traces represent the combined extracted ion chromatograms for the expected [M-H] masses of $\mathrm{Br}_{3} \mathrm{ClPyr}(333.7 \mathrm{~m} / \mathrm{z}), \mathrm{Br}_{2} \mathrm{ClPyr}(255.8 \mathrm{~m} / \mathrm{z})$, and $\mathrm{Br}_{3} \operatorname{Pyr}(302.8 \mathrm{~m} / \mathrm{z})$. 


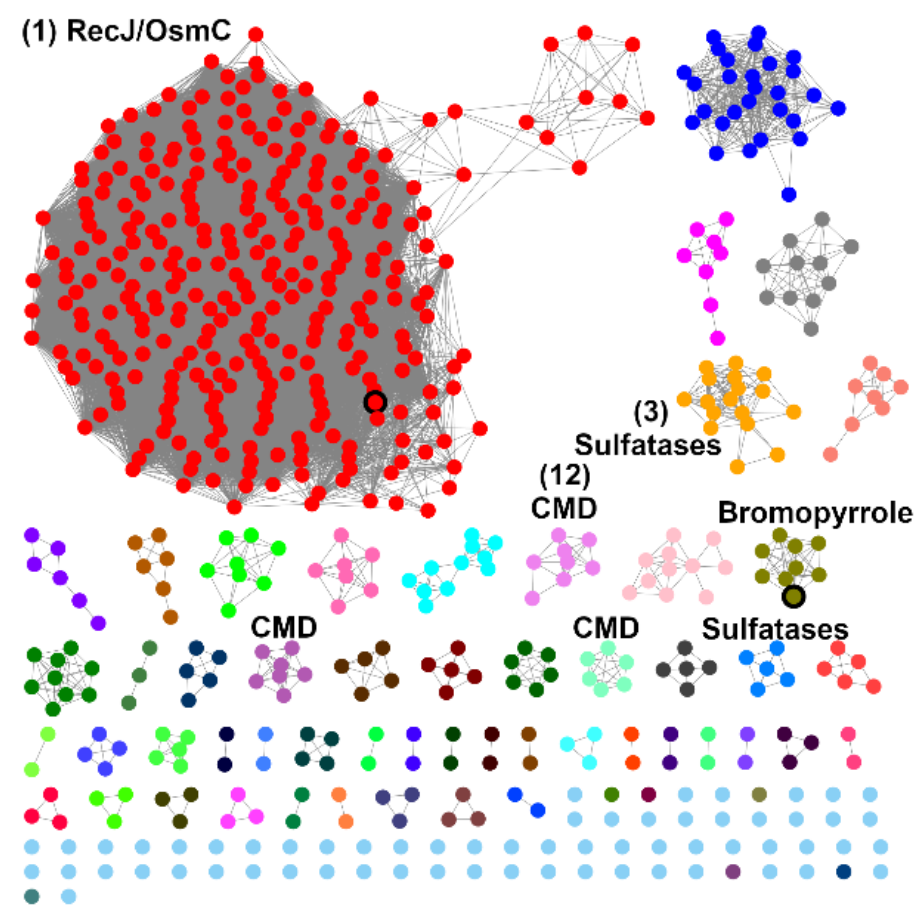

\section{Cluster 1 (RecJ/OsmC) Examples}

Query UniProt ID: A0A2T1N6U8; Mesoflavibacter sabulilitoris; NCBI Taxon ID: 1520893; ENA ID: PXOT01000027; Cluster: 1

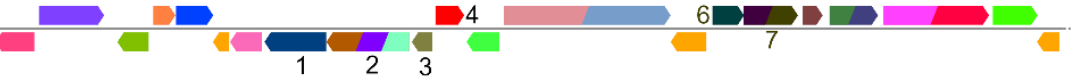

Query UniProt ID: A0A0N0CFF6; Polaribacter dokdonensis DSW-5; NCBI Taxon ID: 1300348; ENA ID: LGBR01000001; Cluster: 1 C

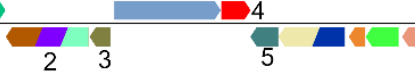

Query UniProt ID: A0A238XLX9; Maribacter sedimenticola; NCBI Taxon ID: 228956; ENA ID: FZNV01000003; Cluster: 1

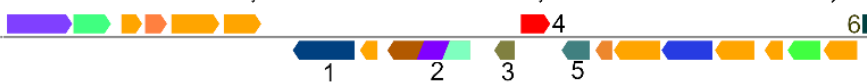

Query UniProt ID: A0A1H9BAJ1; Hyunsoonleella jejuensis; NCBI Taxon ID: 419940; ENA ID: FOFN01000001; Cluster: 1
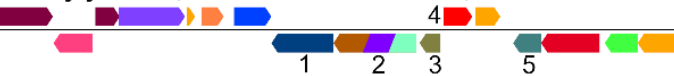

6

Query UniProt ID: A0A162ZBF3; Aquimarina aggregata; NCBI Taxon ID: 1642818; ENA ID: LQRT01000024; Cluster: 1 D $\mathrm{C}$
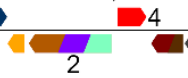

MFS Transporter (PF05977)

Thymidine kinase (PF00265)

2 Exonuclease RecJ (PF01368)

Exonuclease RecJ (PF02272)

Exonuclease RecJ (PF17768)

Alanine racemase (PF00842)

Alanine racemase (PF01168)

3 OsmC family protein (PF02566)

4 Bmp8 Homolog (PF02627)

5 Uracil-DNA glycosylase (PF03167) 


\section{Cluster 3 (Sulfatase) Examples}

Query UniProt ID: A0A1Z9R3X1; Candidatus Puniceispirillum sp. TMED245; NCBI Taxon ID: 1986635; ENA ID: NHKQ01000089; Cluster: 3

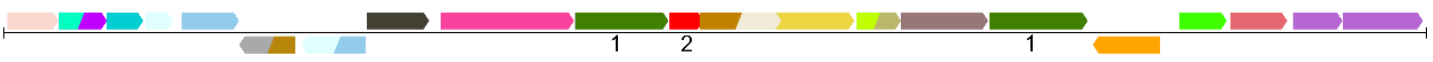

Query UniProt ID: A0A2E7PMC2; Rhodospirillaceae bacterium; NCBI Taxon ID: 1898112; ENA ID: PBPM01000102; Cluster: 3

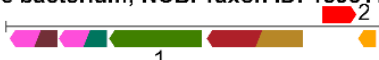

Query UniProt ID: A0A2D8PUY2; Rhodospirillaceae bacterium; NCBI Taxon ID: 1898112; ENA ID: NZOD01000157; Cluster: 3

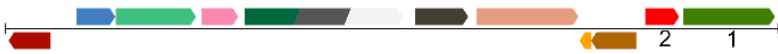

1 Sulfatase (PF00884)

2 Bmp8 homolog (PF02627)

\section{Cluster 10 (Bromopyrrole)}

Query UniProt ID: A0A161ZT39; Pseudoalteromonas luteoviolacea DSM 6061; NCBI Taxon ID: 1365250; ENA ID: AUYB01000136; Cluster: 10

Query UniProt ID: A0A166ZKI4; Pseudoalteromonas luteoviolacea NCIMB 1942; NCBI Taxon ID: 1365253; ENA ID: AUXT01000190; Cluster: 10

Query UniProt ID: A0A16ZZKI4; Pseudoalteromonas luteoviolacea NCIMB 1942; NCBITaxon ID: 1365253; ENA ID: AUXT01000190; Cluster: 10

Query UniProt ID: A0A162C362; Pseudoalteromonas luteoviolacea S4060-1; NCBI Taxon ID: 1365257; ENA ID: AUXX01000045; Cluster: 10

Query UniProt ID: A0A1C0TW18; Pseudoalteromonas luteoviolacea; NCBI Taxon ID: 43657; ENA ID: MAUJ01000001; Cluster: 10

Q............

Query UniProt ID: A0A0F6AI40; Pseudoalteromonas /uteoviolacea S4054; NCBI Taxon ID: 1129367; ENA ID: AUXW01000001; Cluster: 10

Query UniProt ID: A0A167LKE8; Pseudoalteromonas luteoviolacea CPMOR-1; NGBITaxon ID: 1365248; ENA ID: AUYC01000020; Clusters 10

Query UniProt ID: V4HM79; Pseudoalteromonas luteoviolacea 2ta16; NCBI Taxon ID: 1353533; ENA ID: AUSV01000133; Cluster: 10

Query UniProt ID: V4HM79; Pseudoalteromonas luteoviolacea 2ta16; NCBI Taxon ID: 1353533; ENA ID: AUSV01000133; Cluster: 10

Query UniProt ID: A0A0S2JY69; Pseudoalteromonas phenolica; NCBI Taxon ID: 161398; ENA ID: CP013187; Cluster: 10

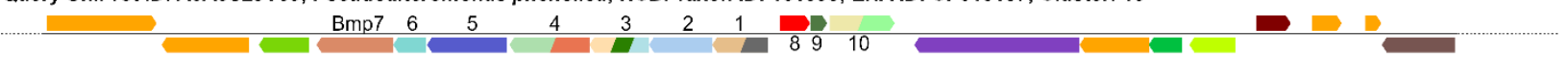

Query UniProt ID: U6BHC9; Pseudoalteromonas phenolica O-BC30; NCBI Taxon ID: 1315281; ENA ID: KF540211; Cluster: 10

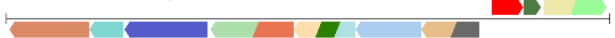

Query UniProt ID: A0A2D5SZN8; Pseudoalteromonas sp; NCBI Taxon ID: 53249; ENA ID: NYXN01000072; Cluster: 10

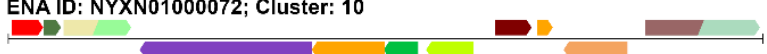

\section{Cluster 12 (CMD) Example}

Query UniProt ID: A0A2E5CH83; Woeseiaceae bacterium; NCBI Taxon ID: 2026806; ENA ID: PBAE01000011; Cluster: 12

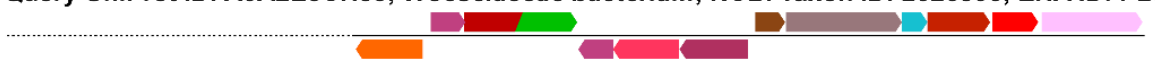

Methylenetetrahydrofolate reductase (PF02219)

Regulator (PF01047)

4-hydroxyphenylacetate 3-monooxygenase (PF11794)

4-hydroxyphenylacetate 3-monooxygenase (PF03241)

Regulator (PF01047)

2-hydroxyhepta-2,4-diene-1,7-dioate isomerase (PF01557)

4-hydroxy-tetrahydrodipicolinate synthase (PF00701)

5-carboxymethyl-2-hydroxymuconate isomerase (PF02962)

5-carboxymethyl-2-hydroxymuconate semialdehyde dehydrogenase (PF00171)
Aromatic ring-opening dioxygenase subunit LigA (PF07746)

Extradiol ring-cleavage dioxygenase (PF02900)

Bmp8 homolog (PF02627)

4-hydroxybenzoate transporter (PF07690) 


\section{PLCMD Gene Cluster}

Query UniProt ID: V4HUK3; Pseudoalteromonas luteoviolacea 2ta16; NCBI Taxon ID: 1353533; ENA ID: AUSV01000036; Cluster: 1

$\begin{array}{ll} & \\ \text { Histidine kinase (PF02518) } & \text { Extracellular nuclease (PF03372) } \\ \text { Uncharacterized (PF08811) } & \text { Extracellular nuclease (PF04151) } \\ \text { Uncharacterized (PF07394) } & \text { Cytochrome C peroxidase (PF03150) } \\ \text { Uncharacterized (IPR036465) } & \text { Regulator (PF12802) } \\ \text { Chemotaxis protein (PF00015) } & \text { Regulator (PF12802) } \\ \text { Chemotaxis protein (PF08376) } & \text { Cytochrome oxidase (PF01654) } \\ \text { Uncharacterized (PF08856) } & \text { Cytochrome oxidase (PF01654) } \\ \text { Uncharacterized (none) } & \text { Cytochrome oxidase (PF02322) } \\ \text { Extracellular nuclease (PF00932) } & \end{array}$

Transglycosylase (PF13406)

YcgL domain (PF05166)

Septum site protein (PF03775)

Septum site protein (PF05209)

Site determining protein (PF13614)

Cell division factor (PF03776)

Figure S8. Example biosynthetic genes clusters of different Bmp8 homologs. Annotations and PFAMs were obtained from the genome neighborhood network gene cluster diagrams. ${ }^{2}$ 


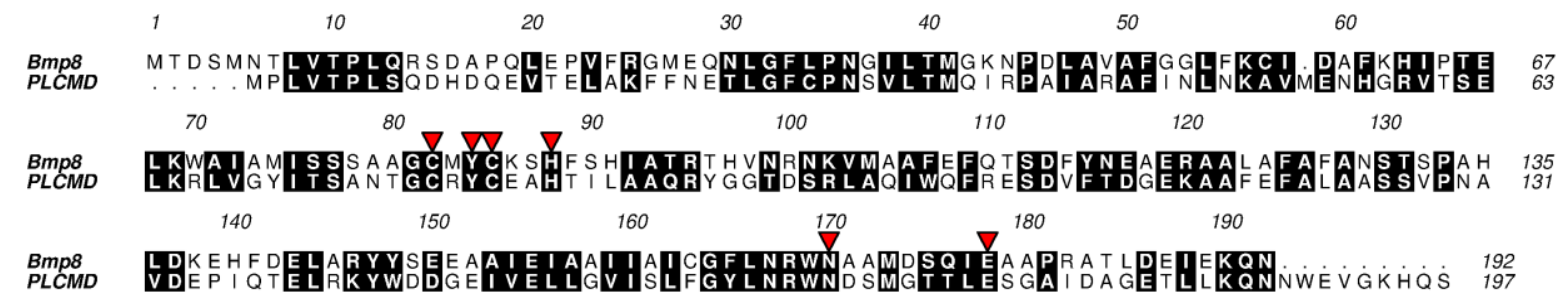

Figure S9. Sequence alignment of Bmp8 and PLCMD. Proposed catalytic residues are indicated. 


\section{Cartesian coordinates, energies, and frequencies}

1. For all possible debromination mechanisms of 2,3,4,5-tetrabromopyrrole substrate.

\section{Starting Materials}

\section{2,3,4,5-tetrabromopyrrole}

C

C

C

C

N

$\mathrm{Br}$

$\mathrm{Br}$

$\mathrm{Br}$

$\mathrm{H}$

$\mathrm{Br}$

$$
\begin{array}{llll}
\text { Thiolate } & & & \\
\text { C } & 1.13752600 & -0.00017000 & -0.00003400 \\
\text { S } & -0.71668900 & 0.00002500 & 0.00000800 \\
\text { H } & 1.54737200 & 0.59208500 & -0.82923300 \\
\text { H } & 1.54753200 & 0.42268200 & 0.92706100 \\
\text { H } & 1.54696800 & -1.01414100 & -0.09774200
\end{array}
$$

\section{Intermediates}

\section{2,3,4-tribromo-5-(methylthio)-1 H-pyrrole}

C

$$
\begin{array}{rrr}
-1.11490400 & -0.84482200 & -0.08056700 \\
-0.49007900 & 0.38691500 & -0.03396500 \\
0.90174700 & 0.13675900 & -0.15863400 \\
1.09407100 & -1.23287300 & -0.26886700 \\
-0.16170900 & -1.80927100 & -0.21604400 \\
-2.98405000 & -1.28968400 & -0.00227900 \\
-1.35793600 & 2.09873800 & 0.15524300 \\
2.31068900 & 1.45782200 & -0.14435600 \\
-0.33711700 & -2.80271900 & -0.27620100 \\
2.54554800 & -2.19967500 & -0.46425800 \\
3.22705000 & -2.15044100 & 1.24453900 \\
4.15307100 & -2.72949100 & 1.22212000 \\
3.44748500 & -1.12016400 & 1.52822200 \\
2.52786100 & -2.60177300 & 1.94995800
\end{array}
$$

(S)-2,3,4,5-tetrabromo-2H-pyrrole

$\begin{array}{llll}\mathrm{C} & -1.07488200 & -0.88693100 & 0.33926200 \\ \mathrm{C} & -0.55495100 & 0.48068500 & 0.11979900 \\ \mathrm{C} & 0.76485000 & 0.40299400 & 0.37370400 \\ \mathrm{C} & 1.05721900 & -1.01745900 & 0.77437900 \\ \mathrm{~N} & -0.19868900 & -1.74935200 & 0.69885900 \\ \mathrm{Br} & -2.89283700 & -1.32206000 & 0.10616900 \\ \mathrm{Br} & -1.55356900 & 1.96508100 & -0.40733800 \\ \mathrm{Br} & 2.04123200 & 1.75351800 & 0.30316800 \\ \mathrm{Br} & 2.36899800 & -1.84000200 & -0.46773200 \\ \mathrm{H} & 1.50357300 & -1.10906000 & 1.76576500\end{array}$

(R)-3,4,5-tribromo-2-(methylthio)-2H-pyrrole 


\begin{tabular}{lrrrllll}
$\mathrm{C}$ & 1.11672300 & 0.75879600 & -0.40371800 & $\mathrm{Br}$ & 3.03110500 & -0.37601000 & 0.00007500 \\
$\mathrm{C}$ & 0.26528600 & -0.42428900 & -0.17355300 & $\mathrm{H}$ & -1.32597500 & -2.92544600 & -0.00000900 \\
$\mathrm{C}$ & -0.98804600 & -0.01828900 & -0.45261100 & & & & \\
$\mathrm{C}$ & -0.92741700 & 1.43768800 & -0.84800500 & \multicolumn{2}{l}{ Bromide ion } & & \\
$\mathrm{N}$ & 0.49464800 & 1.80896000 & -0.78570200 & $\mathrm{Br}$ & 0.00000000 & 0.00000000 & 0.00000000 \\
$\mathrm{Br}$ & 2.98974900 & 0.72089100 & -0.14301900 & & & & \\
$\mathrm{Br}$ & 0.84436200 & -2.10367300 & 0.40571400 & Methyl hypobromothioite & \\
$\mathrm{Br}$ & -2.56160000 & -1.00559600 & -0.32271600 & $\mathrm{~S}$ & 1.11339000 & -0.73515000 & -0.00000200 \\
$\mathrm{H}$ & -1.29224700 & 1.59283900 & -1.86923200 & $\mathrm{C}$ & 2.06727900 & 0.81580000 & 0.00002300 \\
$\mathrm{~S}$ & -1.96588500 & 2.54228700 & 0.19310400 & $\mathrm{H}$ & 1.87267000 & 1.40055900 & 0.90001900 \\
$\mathrm{C}$ & -1.37418200 & 2.04220100 & 1.84959300 & $\mathrm{H}$ & 3.11334800 & 0.48844200 & -0.00086100 \\
$\mathrm{H}$ & -1.84904600 & 2.71796700 & 2.56306000 & $\mathrm{H}$ & 1.87164900 & 1.40146000 & -0.89923400 \\
$\mathrm{H}$ & -1.66908300 & 1.01398900 & 2.06933200 & $\mathrm{Br}$ & -1.05930200 & 0.10220400 & -0.00000100 \\
$\mathrm{H}$ & -0.29003700 & 2.15246900 & 1.91762300 & & & &
\end{tabular}

\section{2,3,4-tribromopyrrol-1-ide}

$\begin{array}{llll}\mathrm{C} & -1.09152400 & -0.89398100 & -0.00001900 \\ \mathrm{C} & -0.00418400 & -0.02160600 & 0.00003300 \\ \mathrm{C} & 1.11642900 & -0.88565000 & 0.00002800 \\ \mathrm{C} & 0.62762100 & -2.18812400 & 0.00024300 \\ \mathrm{~N} & -0.74146600 & -2.18314000 & -0.00013200 \\ \mathrm{H} & 1.19407800 & -3.11117100 & 0.00038700 \\ \mathrm{Br} & -3.00607600 & -0.36562800 & -0.00008500 \\ \mathrm{Br} & -0.00330100 & 1.93113100 & 0.00004100 \\ \mathrm{Br} & 3.01240900 & -0.35609400 & 0.00001000\end{array}$

\section{Transition states}

\section{$S_{N} A R$ at $C 5$ position}

(1)

C

$\begin{array}{lllll}\text { C } & 0.94782500 & 0.45185600 & -0.11762200\end{array}$

$\begin{array}{lllll}\text { C } & -0.47570200 & 0.59080100 & -0.16375000\end{array}$

$\begin{array}{lllll}C & -1.02792200 & -0.68494800 & -0.11737700\end{array}$

$\begin{array}{lllll}\mathrm{N} & 0.02430600 & -1.58267100 & -0.23870800\end{array}$

$\mathrm{Br} \quad 2.91139200 \quad-1.82863200-0.22113800$

$\mathrm{Br} \quad 2.24941900 \quad 1.88745400 \quad-0.13907200$

$\mathrm{Br} \quad-1.47311900 \quad 2.24900000 \quad-0.08800700$

$\begin{array}{llll}\mathrm{H} & -0.36796800 & 0.52513100 & 2.38938500\end{array}$

H $\quad-1.08032600 \quad 0.11877500 \quad 3.96005800$

H $\quad-0.08268400 \quad-2.52150900 \quad 0.11968100$

$\mathrm{Br} \quad-2.74190700 \quad-1.18948500-1.19881100$

$\mathrm{H} \quad 0.18988100 \quad-0.91257000 \quad 3.26572900$

\section{Complexes}




\section{2,3,4,5-tetrabromopyrrole and thiolate}

$\begin{array}{llll}\mathrm{C} & 0.00000000 & 0.00000000 & 0.00000000 \\ \mathrm{~S} & 0.00000000 & 0.00000000 & 1.84657558 \\ \mathrm{C} & 7.04351348 & 0.00000000 & 2.90868038 \\ \mathrm{C} & 7.22816262 & 0.31354069 & 1.57966547 \\ \mathrm{C} & 5.92932687 & 0.40790201 & 1.00074749 \\ \mathrm{C} & 5.00246666 & 0.15130191 & 1.98618397 \\ \mathrm{~N} & 5.69718115 & -0.09554708 & 3.14326848 \\ \mathrm{Br} & 8.33225608 & -0.28669470 & 4.32029383 \\ \mathrm{Br} & 8.93362544 & 0.57060127 & 0.69612865 \\ \mathrm{Br} & 5.52306684 & 0.82412431 & -0.85030275 \\ \mathrm{H} & 0.51388644 & -0.88082502 & -0.40094850 \\ \mathrm{H} & -1.02661349 & -0.00673229 & -0.38468016 \\ \mathrm{H} & 5.27237199 & -0.31539681 & 4.03265139 \\ \mathrm{Br} & 2.96094362 & 0.09334663 & 1.96877066 \\ \mathrm{H} & 0.50206617 & 0.88786634 & -0.40013072\end{array}$

(S)-2,3,4,5-tetrabromo-2 $H$-pyrrole and thiolate

$\begin{array}{llll}\mathrm{C} & 4.49859100 & -0.48167100 & 1.30202800 \\ \mathrm{~S} & 4.56865500 & 1.34615400 & 1.03873000 \\ \mathrm{C} & -1.56071200 & 0.98398400 & -0.43719700 \\ \mathrm{C} & -1.31102400 & -0.42710700 & -0.14511000 \\ \mathrm{C} & -0.11065700 & -0.69255100 & -0.70973100 \\ \mathrm{C} & 0.41245400 & 0.57547200 & -1.30481400 \\ \mathrm{~N} & -0.63985000 & 1.57106000 & -1.11312700 \\ \mathrm{Br} & -3.12920600 & 1.88540800 & 0.14691400 \\ \mathrm{Br} & -2.41332600 & -1.57561400 & 0.84518900 \\ \mathrm{Br} & 0.84851900 & -2.29483300 & -0.67633600 \\ \mathrm{H} & 4.45184700 & -0.73143500 & 2.36826500 \\ \mathrm{H} & 3.60749000 & -0.90425200 & 0.82044600 \\ \mathrm{H} & 5.37730000 & -0.98152400 & 0.87841100 \\ \mathrm{Br} & 1.99995600 & 1.12342800 & -0.39468500 \\ \mathrm{H} & 0.66390000 & 0.48892400 & -2.36380300\end{array}$

\section{Products}

\section{2,3,4-tribromo-1H-pyrrole}

$0.65387200-2.15499900 \quad 0.00026200$

$\begin{array}{llll}\text { C } & 1.12405500 & -0.86084300 & 0.00010000\end{array}$

$\begin{array}{llll}\text { C } & 0.00237900 & 0.01644400 & 0.00016200\end{array}$

$\begin{array}{llll}\text { C } & -1.12164900 & -0.78380600 & 0.00006800\end{array}$

$\begin{array}{llll}N & -0.71777600 & -2.09122200 & -0.00021000\end{array}$

$\begin{array}{llll}\mathrm{Br} & 2.93514100 & -0.34532800 & -0.00006600\end{array}$

$\begin{array}{llll}\mathrm{Br} & 0.03115200 & 1.89167700 & 0.00003100\end{array}$

$\begin{array}{llll}\mathrm{H} & -1.34354800 & -2.88293400 & 0.00039400\end{array}$

$\mathrm{Br} \quad-2.93106000 \quad-0.30878600-0.00004500$

$\begin{array}{llll}H & 1.18288300 & -3.09399100 & 0.00033900\end{array}$

\section{Water}

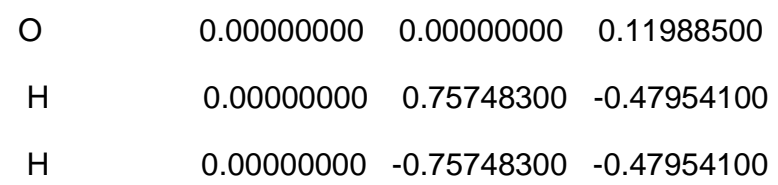


Table S3. Energies from geometry optimization and single point calculations using B3LYP-D3 for all possible debromination mechanisms of 2,3,4,5-tetrabromopyrrole substrate.

\begin{tabular}{|c|c|c|c|c|c|}
\hline \multirow[b]{2}{*}{ Structures } & \multicolumn{4}{|c|}{ Geometry optimizations } & \multirow{2}{*}{$\begin{array}{c}\begin{array}{c}\text { Single } \\
\text { point } \\
\text { calc. }\end{array} \\
\mathbf{H}\end{array}$} \\
\hline & ZPVE & E & $\mathbf{H}$ & G & \\
\hline \multicolumn{6}{|c|}{ Starting Material } \\
\hline 2,3,4,5-tetrabromopyrrole & -260.3939 & -260.3521 & -260.3411 & -260.3921 & -261.2504 \\
\hline Thiolate & -438.1987 & -438.1620 & -438.1580 & -438.1860 & -438.2080 \\
\hline Hydronium ion & -76.7961 & -76.7630 & -76.7592 & -76.7804 & -76.7850 \\
\hline \multicolumn{6}{|c|}{ Intermediates } \\
\hline 2,3,4-tribromo-5-(methylthio)-1H-pyrrole & -685.3516 & -685.2708 & -685.2579 & -685.3128 & -685.9906 \\
\hline (S)-2,3,4,5-tetrabromo-2H-pyrrole & -260.3831 & -260.3420 & -260.3314 & -260.3821 & -261.2418 \\
\hline$(R)$-3,4,5-tribromo-2-(methylthio)-2H-pyrrole & -685.3334 & -685.2534 & -685.2409 & -685.2950 & -685.9759 \\
\hline 2,3,4-tribromopyrrol-1-ide & -247.3475 & -247.3089 & -247.2996 & -247.3458 & -248.006 \\
\hline 3,4,5-tribromo-1 $\mathrm{H}$-pyrrol-2-ide & -247.2839 & -247.2456 & -247.2361 & -247.2828 & -247.9502 \\
\hline Bromide ion & -13.3209 & -13.3209 & -13.3186 & -13.3371 & -13.5721 \\
\hline Methyl hypobromothioite & -451.2642 & -451.2254 & -451.2199 & -451.2542 & -451.4738 \\
\hline \multicolumn{6}{|c|}{ Transition States } \\
\hline 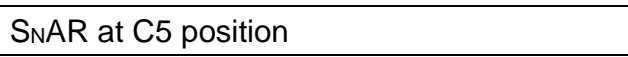 & -698.5994 & -698.5214 & -698.5063 & -698.5692 & -699.4489 \\
\hline \multicolumn{6}{|c|}{ Complexes } \\
\hline $\begin{array}{l}\text { 2,3,4,5-tetrabromopyrrole } \\
\text { and thiolate }\end{array}$ & -698.6042 & -698.5254 & -698.5092 & -698.5746 & -699.4666 \\
\hline $\begin{array}{l}(\mathrm{S})-2,3,4,5 \text {-tetrabromo- } 2 \mathrm{H} \text {-pyrrole } \\
\text { and thiolate }\end{array}$ & -698.5832 & -698.5042 & -698.4894 & -698.5542 & -699.4534 \\
\hline \multicolumn{6}{|c|}{ Products } \\
\hline 2,3,4-tribromo-1 $\mathrm{H}$-pyrrole & -247.8440 & -247.7917 & -247.7824 & -247.8285 & -248.4783 \\
\hline Water & -76.4250 & -76.4036 & -76.3999 & -76.4213 & -76.4392 \\
\hline
\end{tabular}

ZPVE = zero-point vibrational energy; $E$ = electronic energy; $\mathrm{H}=$ enthalpy; $\mathrm{G}=$ Gibbs free energy. 
Table S4. Energies from geometry optimization and single point calculations using M06-2X for all possible debromination mechanisms of 2,3,4,5-tetrabromopyrrole substrate.

\begin{tabular}{|c|c|c|c|c|c|}
\hline \multirow[b]{2}{*}{ Structures } & \multicolumn{4}{|c|}{ Geometry optimizations } & \multirow{2}{*}{$\begin{array}{c}\begin{array}{c}\text { Single } \\
\text { point } \\
\text { calc. }\end{array} \\
\mathbf{H} \\
\end{array}$} \\
\hline & ZPVE & $\mathbf{E}$ & H & G & \\
\hline \multicolumn{6}{|c|}{ Starting Material } \\
\hline 2,3,4,5-tetrabromopyrrole & -260.1224 & -260.0795 & -260.0687 & -260.1194 & -260.9783 \\
\hline Thiolate & -438.1322 & -438.0953 & -438.0913 & -438.1189 & -438.1422 \\
\hline Hydronium ion & -76.7593 & -76.7261 & -76.7223 & -76.7435 & -76.7478 \\
\hline \multicolumn{6}{|c|}{ Intermediates } \\
\hline 2,3,4-tribromo-5-(methylthio)- $1 \mathrm{H}$-pyrrole & -685.0644 & -684.9820 & -684.9693 & -685.0238 & -685.7047 \\
\hline (S)-2,3,4,5-tetrabromo-2H-pyrrole & -260.1069 & -260.0646 & -260.0541 & -260.1046 & -260.9660 \\
\hline$(R)$-3,4,5-tribromo-2-(methylthio)-2H-pyrrole & -685.0432 & -684.9617 & -684.9493 & -685.0034 & -685.6892 \\
\hline 2,3,4-tribromopyrrol-1-ide & -247.1276 & -247.0876 & -247.0786 & -247.1243 & -247.7842 \\
\hline 3,4,5-tribromo-1 $\mathrm{H}$-pyrrol-2-ide & -247.0629 & -247.0233 & -247.0140 & -247.0600 & -247.7279 \\
\hline Bromide ion & -13.2702 & -13.2702 & -13.2678 & -13.2863 & -13.4956 \\
\hline Methyl hypobromothioite & -451.1451 & -451.1057 & -451.1003 & -451.1344 & -451.3589 \\
\hline \multicolumn{6}{|c|}{ Transition States } \\
\hline $\mathrm{S}_{\mathrm{NAR}}$ at $\mathrm{C5}$ position & -698.2429 & -698.1631 & -698.1481 & -698.2089 & -699.1102 \\
\hline \multicolumn{6}{|c|}{ Complexes } \\
\hline $\begin{array}{l}\text { 2,3,4,5-tetrabromopyrrole } \\
\text { and thiolate }\end{array}$ & -698.2636 & -698.1826 & -698.1669 & -698.2309 & -699.1282 \\
\hline $\begin{array}{l}\text { (S)-2,3,4,5-tetrabromo-2H-pyrrole } \\
\text { and thiolate }\end{array}$ & -698.2415 & -698.1611 & -698.1468 & -698.2074 & -699.1136 \\
\hline \multicolumn{6}{|c|}{ Products } \\
\hline 2,3,4-tribromo-1 $\mathrm{H}$-pyrrole & -247.6165 & -247.5632 & -247.5540 & -247.5998 & -248.2498 \\
\hline Water & -76.3895 & -76.3678 & -76.3640 & -76.3854 & -76.4014 \\
\hline
\end{tabular}

ZPVE = zero-point vibrational energy; $E$ = electronic energy; $\mathrm{H}=$ enthalpy; $\mathrm{G}=$ Gibbs free energy. 


\section{For dehalogenation of other substrates}

\section{Starting Materials}

\section{2,3,4,5-tetrachloropyrrole}

\begin{tabular}{|c|c|c|c|}
\hline & 1.11816700 & -0.77433900 & -0.00009700 \\
\hline & 0.71448400 & 0.54448500 & 0.00008800 \\
\hline & -0.71450400 & 0.54449000 & 0.00009600 \\
\hline & -1.11816100 & -0.77433000 & 0.00000000 \\
\hline & 0.00000200 & -1.56837800 & 0.00021900 \\
\hline & 0.00000300 & -2.57813600 & 0.00148500 \\
\hline & 1.74008000 & 1.93184900 & 0.00002800 \\
\hline & 2.69703900 & -1.45198800 & -0.00011500 \\
\hline & -1.74009600 & 1.93186600 & -0.00002700 \\
\hline & -2.69701900 & -1.45202500 & -0.00009400 \\
\hline
\end{tabular}

\section{2,3,4-tribromo-5-chloro-1H-pyrrole}

$\begin{array}{llll}\mathrm{C} & -0.94710100 & 1.57574800 & -0.00003200 \\ \mathrm{C} & -1.06834200 & 0.20199000 & 0.00005100 \\ \mathrm{C} & 0.25376000 & -0.33657600 & 0.00021000 \\ \mathrm{C} & 1.12336100 & 0.73170500 & 0.00018300 \\ \mathrm{~N} & 0.38949100 & 1.89021200 & 0.00000700 \\ \mathrm{H} & 0.76900900 & 2.82712700 & 0.00108200 \\ \mathrm{Cl} & -2.14620700 & 2.80606900 & -0.00000300 \\ \mathrm{Br} & -2.71670000 & -0.78070500 & -0.00005500 \\ \mathrm{Br} & 0.73367600 & -2.19505800 & 0.00003300 \\ \mathrm{Br} & 3.03502400 & 0.78150700 & -0.00007900\end{array}$

\section{Intermediates}

\section{(S)-2,3,4,5-tetrachloro-2H-pyrrole}

$\begin{array}{llll}\text { C } & -1.06854200 & -0.79940400 & 0.18994300 \\ \text { C } & -0.58562700 & 0.59381700 & 0.03156700 \\ \text { C } & 0.74243600 & 0.53480700 & 0.24455700 \\ \text { C } & 1.07758000 & -0.90319100 & 0.57436900\end{array}$

$\begin{array}{llll}\mathrm{N} & -0.16522200 & -1.65992000 & 0.47244600 \\ \mathrm{H} & 1.48148900 & -0.99930600 & 1.58565300 \\ \mathrm{Cl} & -1.55548400 & 1.95624400 & -0.34412500 \\ \mathrm{Cl} & -2.73359100 & -1.22352400 & 0.00428500 \\ \mathrm{Cl} & 2.32628800 & -1.59319500 & -0.54361100 \\ \mathrm{Cl} & 1.88513900 & 1.80533200 & 0.22842700\end{array}$

(S)-3,4,5-tribromo-2-chloro-2 $\mathrm{H}$-pyrrole

$\begin{array}{llll}\mathrm{C} & -1.09874800 & 0.81862700 & -0.21534500 \\ \mathrm{C} & -0.16082500 & -0.32025100 & -0.07262700 \\ \mathrm{C} & 1.06372600 & 0.21191400 & -0.22657400 \\ \mathrm{C} & 0.88416000 & 1.68772500 & -0.50410000 \\ \mathrm{~N} & -0.55341600 & 1.94998900 & -0.43607700 \\ \mathrm{H} & 1.25329600 & 1.95620500 & -1.49773600 \\ \mathrm{Cl} & 1.76257500 & 2.73496800 & 0.67874800 \\ \mathrm{Br} & -3.02461600 & 0.62301700 & -0.07755900 \\ \mathrm{Br} & -0.63707500 & -2.14669600 & 0.25892100 \\ \mathrm{Br} & 2.76246100 & -0.66171200 & -0.20640700\end{array}$

\section{Methyl hypochlorothioite}

$\begin{array}{llll}\text { S } & 0.48918100 & -0.71932200 & -0.00002000 \\ \text { C } & 1.61532300 & 0.71218800 & -0.00005500 \\ \text { H } & 1.48101400 & 1.31521100 & 0.89905200 \\ \text { H } & 2.61889500 & 0.27403700 & 0.00107800 \\ \text { H } & 1.48226300 & 1.31474100 & -0.89961700 \\ \text { Cl } & -1.35888300 & 0.25482600 & 0.00000800\end{array}$

\section{3,4,5-trichloro-1 H-pyrrol-2-ide}

C $\quad-1.09662200-0.57269100 \quad-0.00002500$

$\begin{array}{llll}\text { C } & -0.00367600 & 0.29630900 & 0.00001400\end{array}$ 


$\begin{array}{lrrrlrrr}\mathrm{C} & 1.12054600 & -0.56497600 & 0.00001500 & \mathrm{~N} & 0.92598500 & -1.48893300 & -0.93269800 \\ \mathrm{C} & 0.62416900 & -1.86624300 & 0.00021400 & \mathrm{H} & -4.13850700 & 0.85694500 & 2.17504200 \\ \mathrm{~N} & -0.74341600 & -1.86669500 & -0.00012500 & \mathrm{H} & -3.28989500 & 1.22773100 & 0.66871100 \\ \mathrm{H} & 1.19358700 & -2.78813100 & 0.00035300 & \mathrm{H} & -5.04587400 & 1.42128700 & 0.76542600 \\ \mathrm{Cl} & 2.81049500 & -0.07134500 & 0.00000900 & \mathrm{H} & -0.03167800 & -0.24185800 & -2.33044300 \\ \mathrm{Cl} & -0.00311300 & 2.04697700 & 0.00001900 & \mathrm{Cl} & -1.56068400 & -0.73181900 & -0.61895200 \\ \mathrm{Cl} & -2.79892200 & -0.08736200 & -0.00007400 & \mathrm{Cl} & 0.00525600 & 2.42706300 & -0.68405000 \\ & & & & \mathrm{Cl} & 2.87318600 & 1.36901000 & 1.00242900 \\ & & & \mathrm{Cl} & 3.14216100 & -1.99355300 & 0.44630200\end{array}$

\section{2,3,4,5-tetrachloropyrrole and thiolate}

$\begin{array}{lrrr}\mathrm{C} & -4.87792500 & 0.94197600 & -0.08837300 \\ \mathrm{~S} & -4.99043600 & -0.90376100 & 0.01200700 \\ \mathrm{C} & 2.14513600 & -0.96522100 & -0.00568300 \\ \mathrm{C} & 2.06113000 & 0.41086400 & -0.00103100 \\ \mathrm{C} & 0.67138400 & 0.74237300 & 0.01543700 \\ \mathrm{C} & -0.03634700 & -0.44257400 & 0.02028800 \\ \mathrm{~N} & 0.87093800 & -1.47248200 & 0.00734200 \\ \mathrm{H} & -5.41046800 & 1.33513400 & -0.96382600 \\ \mathrm{H} & -5.31021300 & 1.42479600 & 0.79744000 \\ \mathrm{H} & 0.63014900 & -2.45311500 & 0.00709500 \\ \mathrm{H} & -3.83540200 & 1.27579100 & -0.16392100 \\ \mathrm{Cl} & -1.74199600 & -0.73985900 & 0.03610600 \\ \mathrm{Cl} & -0.00067200 & 2.33565800 & 0.02655100 \\ \mathrm{Cl} & 3.38743300 & 1.52034600 & -0.01296600 \\ \mathrm{Cl} & 3.52559300 & -1.99494300 & -0.02405200\end{array}$

\section{(S)-2,3,4,5-tetrachloro-2H-pyrrole and thiolate}

$\begin{array}{llll}\text { C } & -4.20675900 & 0.78963200 & 1.08223800 \\ \text { S } & -4.41544300 & -0.95683200 & 0.51512200 \\ \text { C } & 1.86355300 & -1.00720500 & -0.20496400 \\ \text { C } & 1.77793900 & 0.43905400 & 0.05276600 \\ \text { C } & 0.66625200 & 0.84761000 & -0.59633600 \\ \text { C } & 0.04110200 & -0.35812000 & -1.24523500\end{array}$

\begin{tabular}{lrrr}
\multicolumn{4}{l}{$\begin{array}{l}\text { 2,3,4-tribromo-5-chloro-1H-pyrrole } \\
\text { thiolate }\end{array}$} \\
$\mathrm{C}$ & -5.66809300 & 0.29876800 & 0.00000100 \\
$\mathrm{~S}$ & -5.60325500 & -1.55140200 & 0.00000100 \\
$\mathrm{C}$ & 1.47587000 & -1.07965500 & -0.00000100 \\
$\mathrm{C}$ & 1.30533600 & 0.28665000 & 0.00000000 \\
$\mathrm{C}$ & -0.10001800 & 0.52688100 & -0.00000100 \\
$\mathrm{C}$ & -0.73277900 & -0.69919200 & -0.00000100 \\
$\mathrm{~N}$ & 0.23877700 & -1.66934700 & -0.00000100 \\
$\mathrm{H}$ & -6.19089000 & 0.68727600 & 0.88342500 \\
$\mathrm{H}$ & -4.66069700 & 0.73356200 & 0.00000800 \\
$\mathrm{H}$ & 0.05891700 & -2.66342100 & -0.00000100 \\
$\mathrm{H}$ & -6.19087900 & 0.68727700 & -0.88342800 \\
$\mathrm{Br}$ & 3.08317800 & -2.14133700 & 0.00000100 \\
$\mathrm{Br}$ & 2.71015300 & 1.61279500 & 0.00000000 \\
$\mathrm{Br}$ & -0.98382200 & 2.24481600 & 0.00000000 \\
$\mathrm{Cl}$ & -2.41473500 & -1.11806700 & -0.00000200
\end{tabular}

(S)-3,4,5-tribromo-2-chloro-2H-pyrrole and thiolate

$\begin{array}{llll}\text { C } & -4.54814000 & 0.10118500 & 1.15640900 \\ \text { S } & -4.74067800 & -1.72792300 & 0.97003500 \\ \text { C } & 1.44435700 & -1.00091600 & -0.44476400 \\ \text { C } & 1.06183800 & 0.39018500 & -0.15530300\end{array}$




\begin{tabular}{lrrrrllll}
$\mathrm{C}$ & -0.15865400 & 0.54471600 & -0.70797600 & \multicolumn{4}{l}{ Products } \\
$\mathrm{C}$ & -0.54931400 & -0.76669900 & -1.33525000 & $\mathbf{2}, \mathbf{3}$-4-trichloro-1H-pyrrole \\
$\mathrm{N}$ & 0.58262800 & -1.67923500 & -1.10644600 & $\mathrm{C}$ & 0.65133200 & -1.84691400 & 0.00020100 \\
$\mathrm{H}$ & -4.46442400 & 0.39321900 & 2.21074400 & $\mathrm{C}$ & 1.12941000 & -0.55563500 & 0.00008100 \\
$\mathrm{H}$ & -3.64706400 & 0.46455600 & 0.64496300 & $\mathrm{C}$ & 0.01134200 & 0.33017700 & 0.00013800 \\
$\mathrm{H}$ & -5.40394500 & 0.64274300 & 0.73404000 & $\mathrm{C}$ & -1.11758600 & -0.46600600 & 0.00006000 \\
$\mathrm{H}$ & -0.73476900 & -0.67364000 & -2.40884200 & $\mathrm{~N}$ & -0.71998300 & -1.77497700 & -0.00028500 \\
$\mathrm{Cl}$ & -1.97465800 & -1.46760700 & -0.57714500 & $\mathrm{H}$ & -1.34929900 & -2.56382200 & 0.00030700 \\
$\mathrm{Br}$ & -1.26623400 & 2.04166300 & -0.67809200 & $\mathrm{H}$ & 1.17505100 & -2.78877100 & 0.00027500 \\
$\mathrm{Br}$ & 2.06563100 & 1.63838900 & 0.81249300 & $\mathrm{Cl}$ & 0.05073700 & 2.05717300 & 0.00000800 \\
$\mathrm{Br}$ & 3.08892600 & -1.73968000 & 0.14492600 & $\mathrm{Cl}$ & 2.79947700 & -0.08990900 & -0.000007100 \\
& & & & $\mathrm{Cl}$ & -2.78155800 & -0.02563400 & -0.0000230
\end{tabular}

Table S5. Energies from geometry optimization and single point calculations using B3LYP-D3 for dehalogenation of other substrates.

\begin{tabular}{|c|c|c|c|c|c|}
\hline \multirow[b]{2}{*}{ Structures } & \multicolumn{4}{|c|}{ Geometry optimizations } & \multirow{2}{*}{$\begin{array}{c}\begin{array}{c}\text { Single } \\
\text { point } \\
\text { calc. }\end{array} \\
\text { H }\end{array}$} \\
\hline & ZPVE & E & $\mathbf{H}$ & G & \\
\hline \multicolumn{6}{|c|}{ Starting Material } \\
\hline 2,3,4,5-tetrachloropyrrole & -2048.5481 & -2048.5032 & -2048.4934 & -2048.5388 & -2048.6590 \\
\hline 2,3,4-tribromo-5-chloro-1 H-pyrrole & -707.4433 & -707.4007 & -707.3901 & -707.4396 & -708.1030 \\
\hline \multicolumn{6}{|c|}{ Intermediates } \\
\hline (S)-2,3,4,5-tetrachloro-2H-pyrrole & -2048.5338 & -2048.4896 & -2048.4801 & -2048.5252 & -2048.6471 \\
\hline (S)-3,4,5-tribromo-2-chloro-2H-pyrrole & -707.4119 & -707.3701 & -707.3597 & -707.4090 & -708.0896 \\
\hline Methyl hypochlorothioite & -898.2969 & -898.2576 & -898.2523 & -898.2851 & -898.3178 \\
\hline 3,4,5-trichloro-1 $\mathrm{H}$-pyrrol-2-ide & -1588.4604 & -1588.4195 & -1588.4112 & -1588.4529 & -1588.5609 \\
\hline \multicolumn{6}{|c|}{ Complexes } \\
\hline 2,3,4,5-tetrachloropyrrole and thiolate & -2486.7542 & -2486.6722 & -2486.6572 & -2486.7185 & -2486.8702 \\
\hline $\begin{array}{l}(S)-2,3,4,5 \text {-tetrachloro-2H-pyrrole } \\
\text { and thiolate }\end{array}$ & -2486.7387 & -2486.6573 & -2486.6427 & -2486.7047 & -2486.8562 \\
\hline $\begin{array}{l}\text { 2,3,4-tribromo-5-chloro-1 } \mathrm{H} \text {-pyrrole } \\
\text { and thiolate }\end{array}$ & -1145.6314 & -1145.5515 & -1145.5358 & -1145.5999 & -1146.3138 \\
\hline $\begin{array}{l}\text { (S)-3,4,5-tribromo-2-chloro-2H-pyrrole } \\
\text { and thiolate }\end{array}$ & -1145.6105 & -1145.5311 & -1145.5165 & -1145.5785 & -1146.2989 \\
\hline \multicolumn{6}{|c|}{ Products } \\
\hline 2,3,4-trichloro-1H-pyrrole & -1589.0347 & -1589.0347 & -1589.0347 & -1589.0347 & -1589.0347 \\
\hline
\end{tabular}

ZPVE = zero-point vibrational energy; $E$ = electronic energy; $H=$ enthalpy; $G=$ Gibbs free energy. 


\section{For dehalogenation of Dios enzyme.}

\section{Starting Material}

\section{2,6-diiodobenzene-1,4-diol}

$\begin{array}{llll}\text { C } & -0.00517400 & 2.51842900 & 0.00000000\end{array}$

$\begin{array}{llll}\text { C } & -1.21271600 & 1.81411600 & 0.00000000\end{array}$

C $\quad-1.19978400 \quad 0.42210500 \quad 0.00000400$

C $\quad-0.00595200 \quad-0.31743100 \quad 0.00000200$

$\begin{array}{llll}\text { C } & 1.18824400 & 0.42625900 & 0.00000400\end{array}$

O $\quad-0.07797300 \quad-1.67042200 \quad 0.00000000$

$\begin{array}{llll}\text { O } & 0.06464300 & 3.88127900 & -0.00001500\end{array}$

I $\quad-3.07256400 \quad-0.61801100 \quad 0.00000000$

I $\quad 3.07896000 \quad-0.60262400 \quad 0.00000000$

$\begin{array}{llll}H & 2.13242600 & 2.37171500 & 0.00001200\end{array}$

H $\quad-2.15538800 \quad 2.35030100 \quad 0.00000200$

H $\quad 0.81275300 \quad-2.05343900 \quad-0.00003900$

$\begin{array}{llll}\mathrm{H} & -0.82634900 & 4.25669400 & 0.00006900\end{array}$

$\begin{array}{llll}\text { Selenol } & & & \\ \text { Se } & -1.33577800 & -0.49170900 & -0.00000500 \\ \text { C } & -2.03248100 & 1.35023200 & -0.00000200 \\ \text { H } & -1.71071500 & 1.87246800 & -0.89996900 \\ \text { H } & -3.12021600 & 1.23702500 & -0.00062800 \\ \text { H } & -1.71161700 & 1.87159700 & 0.90077100 \\ \text { I } & 1.21045100 & 0.06859700 & 0.00000000\end{array}$

\section{Complex}

\section{2,6-diiodobenzene-1,4-diol and selenol}

$\begin{array}{lllll}\text { C } & -2.85894600 & 1.73947300 & -0.01661900\end{array}$

C $\quad-1.77171300 \quad 2.61349900-0.04812800$

$\begin{array}{llll}\text { C } & -0.47107400 & 2.09497300 & -0.06135800\end{array}$

$\begin{array}{llll}\text { C } & -0.24702400 & 0.72226900 & -0.04334500\end{array}$

$\begin{array}{llll}\text { C } & -1.32413100 & -0.17876700 & -0.01138900\end{array}$

$\begin{array}{llll}\text { C } & -2.61540500 & 0.36753900 & 0.00095400\end{array}$

O $\quad-1.05364000-1.51458400 \quad 0.00514400$

$\begin{array}{lllll}0 & -2.04319600 & 3.95803500 & -0.06495800\end{array}$

$\begin{array}{lllll}\text { I } & 1.87761500 & -0.02698400 & -0.06581200\end{array}$

I $\quad-4.34909100 \quad-0.95634500 \quad 0.04922200$

$\mathrm{H} \quad-3.86543500 \quad 2.13897900 \quad-0.00676400$

H $\quad 0.37715700 \quad 2.77572300 \quad-0.08636400$

$\mathrm{H} \quad-1.88380000 \quad-2.01329700 \quad 0.02862300$

H $\quad-1.20986000 \quad 4.44736300 \quad-0.08903400$

Se $\quad 4.94481100 \quad-0.84428500-0.07057700$

$\begin{array}{llll}\text { C } & 5.51276500 & 0.97064200 & 0.51728200\end{array}$

$\begin{array}{llll}\mathrm{H} & 6.60476200 & 1.02047100 & 0.55970800\end{array}$

$\begin{array}{llll}\text { H } & 5.15546000 & 1.73032300 & -0.18290000\end{array}$

$\begin{array}{llll}\mathrm{H} & 5.11426900 & 1.19716800 & 1.5097510\end{array}$ 
Table S6. Energies from geometry optimization and single point calculations using B3LYP-D3 for Dios enzyme.

\begin{tabular}{|c|c|c|c|c|c|}
\hline \multirow[b]{2}{*}{ Structures } & \multicolumn{4}{|c|}{ Geometry optimizations } & \multirow{2}{*}{$\begin{array}{c}\text { Single } \\
\text { point } \\
\text { calc. } \\
H\end{array}$} \\
\hline & ZPVE & E & H & G & \\
\hline \multicolumn{6}{|c|}{ Starting Material } \\
\hline 2,6-diiodobenzene-1,4-diol & -404.2599 & -404.1723 & -404.1613 & -404.2109 & -404.3372 \\
\hline Selenol & -2439.3853 & -2439.3494 & -2439.3453 & -2439.3734 & -2441.5512 \\
\hline \multicolumn{6}{|c|}{ Complex } \\
\hline 2,6-diiodobenzene-1,4-diol and selenol & -2843.2158 & -2843.0918 & -2843.0754 & -2843.1411 & -2845.8992 \\
\hline
\end{tabular}

ZPVE = zero-point vibrational energy; $E$ = electronic energy; $H=$ enthalpy; $G$ = Gibbs free energy. 


\section{Supplementary References}

(1) Zheng, J., McKinnie, S. M. K., El Gamal, A., Feng, W., Dong, Y., Agarwal, V., Fenical, W., Kumar, A., Cao, Z., Moore, B. S., and Pessah, I. N. (2018) Organohalogens naturally biosynthesized in marine environments and produced as disinfection byproducts alter sarco/endoplasmic reticulum $\mathrm{Ca}^{2+}$ dynamics. Environ. Sci. Technol. 52, 5469-78.

(2) Zallot, R., Oberg, N. O., and Gerlt, J. A. (2018) 'Democratized’ genomic enzymology web tools for functional assignment. Curr. Opin. Chem. Biol. 47, 77-85. 